\title{
Boundary Stabilization of An Anti-Stable Wave Equation With In-Domain Anti-Damping
}

\author{
Andrey Smyshlyaev, Eduardo Cerpa, and Miroslav Krstic
}

\begin{abstract}
We consider the problem of boundary stabilization of a one-dimensional wave equation with an internal spatially varying anti-damping term. This term puts all the eigenvalues of the open-loop system in the right half of the complex plane. We design a feedback law based on the backstepping method and prove exponential stability of the closed-loop system with a desired decay rate. For plants with constant parameters the control gains are found in closed form. Our design also produces a new Lyapunov function for the classical wave equation with passive boundary damping.
\end{abstract}

\section{INTRODUCTION}

The asymptotic stability and stabilization by feedback of wave equations in bounded domains are topics which have been widely studied over the past 30 years. The wave equation being conservative, the main idea is to add some dissipation by means of boundary (e.g. [2], [9]) or distributed (e.g. [4]) damping terms. If the dissipation is large enough, then one expects that the energy of the system is uniformly decreasing. Thus, one expects the solutions to converge polynomially or exponentially to zero. In order to deal with this kind of problem, several tools have been applied. Among them are spectral methods [13], [7], the LQR approach [10], the multiplier technique [5], [11], the microlocal analysis [1], Lyapunov functionals [18], and the Gramian approach [6], [19].

In this paper we are concerned with the stabilization problem of a one-dimensional wave equation with an internal destabilizing term. Because of this term, the system is anti-stable in the sense that the eigenvalues of the openloop system can all be in the right half of the complex plane, which produces an exponential growth of the norm of the solutions. Our objective is to stabilize the string by actuating the position of one of the string's ends (the other, uncontrolled end of the string is pinned).

Our approach is based on the backstepping method which uses a Volterra transformation to map an unstable system into a stable "target" PDE. This method allows us to achieve an arbitrary large exponential decay rate for the closed-loop system. In the framework of infinite-dimensional systems, the backstepping method has been mainly used for parabolic and first-order hyperbolic equations [12], [15], [16]. Recently, in [8] the authors have extended the method in order to deal

Andrey Smyshlyaev and Miroslav Krstic are with the Department of Mechanical and Aerospace Engineering, University of California at San Diego, La Jolla, CA 92093, USA asmyshly@ucsd.edu and krsticlucsd.edu

Eduardo Cerpa is with Departamento de Matemática, Universidad Técnica Federico Santa María, Casilla 110-V, Valparaíso, Chile. eduardo.cerpa@usm.cl with second-order hyperbolic systems. In that paper, they deal with an unstable wave equation. The instability comes from a boundary term of anti-stiffness type which generates a finite number of eigenvalues for the open-loop system in the right half of the complex plane. A more challenging problem has been dealt with in [17] where an infinite number of unstable eigenvalues is generated by the boundary anti-stable term.

To eliminate internal anti-damping and add arbitrary amount of positive damping and stiffness, we develop a novel backstepping transformation. This transformation has a $2 \times 2$ structure, it is invertible, and the kernels of its four Volterra operators are generated from two coupled second order hyperbolic PDEs in Goursat form. For plants with constant coefficients these PDEs can be solved explicitly, resulting in closed-form control gains.

Our design also produces a new Lyapunov function for the classical undamped wave equation with passive boundary damping. This Lyapunov function is "perfect" in the sense that it gives the decay rate exactly equal to the one determined by eigenvalues.

\section{Statement of the Problem and Main Result}

Consider the wave equation

$$
\left\{\begin{array}{l}
u_{t t}=u_{x x}+2 \lambda(x) u_{t}+\alpha(x) u_{x}+\beta(x) u, \\
u(0, t)=0, \quad u(1, t)=U(t), \\
u(x, 0)=u_{0}(x), \quad u_{t}(x, 0)=u_{1}(x),
\end{array}\right.
$$

where for each time $t \geq 0, U(t) \in \mathbb{R}$ is the input and the functions $u(\cdot, t), u_{t}(\cdot, t):[0,1] \rightarrow \mathbb{R}$ form the state of the system. The functions $u_{0}, u_{1}$ are the initial conditions and the functions $\lambda, \alpha, \beta$ are coefficients whose regularity will be defined later. The open-loop plant (i.e. with $U(t)=0$ ) may be unstable depending on the function $\lambda$. For instance, for positive $\lambda(x)$ and $\beta(x)=\alpha(x)=0$, all the eigenvalues of the system are located in the right half of the complex plane. Our objective is to design a feedback law which stabilizes (1) at the origin.

Without loss of generality, we set $\alpha(x) \equiv 0$. Indeed, if $\alpha$ is not identically zero, the following rescaling of the state variable

$$
v(x, t)=e^{\frac{1}{2} \int_{0}^{x} \alpha(\tau) d \tau} u(x, t)
$$

would transform the original wave equation into the one without the first-order spatial derivative term.

Note that for constant $\lambda$, one can eliminate the antidamping term by introducing the new variable $v(x, t)=$ $e^{-\lambda t} u(x, t)$. Then one designs the controller for $v$-system 
that achieves a decay rate larger than $\lambda$. However, this idea does not work for spatially varying $\lambda(x)$.

The main idea of this paper is to use the transformation

$$
\begin{aligned}
w(x, t)= & h(x) u(x, t)-\int_{0}^{x} k(x, y) u(y, t) d y \\
& -\int_{0}^{x} s(x, y) u_{t}(y, t) d y,
\end{aligned}
$$

and the feedback

$$
U(t)=\frac{1}{h(1)} \int_{0}^{1}\left[k(1, y) u(y, t)+s(1, y) u_{t}(y, t)\right] d y,
$$

where the function $h=h(x)$ and kernels $k=k(x, y)$ and $s=s(x, y)$ are appropriately chosen, to convert the original system (1) into the following one

$$
\left\{\begin{array}{l}
w_{t t}=w_{x x}-2 d(x) w_{t}-c(x) w \\
w(0, t)=0, \quad w(1, t)=0 \\
w(x, 0)=w_{0}(x), \quad w_{t}(x, 0)=w_{1}(x)
\end{array}\right.
$$

with appropriate functions $d=d(x)$ and $c=c(x)$ so that this new system is exponentially stable. The functions $d$ and $c$ can always be chosen to provide any desired decay rate.

Then, we use exponential stability of (4) and the invertibility of the transformation (2) to obtain stability of the closedloop system (1) and (3).

Introducing the space $H_{L}^{1}(0,1)$ defined by

$$
H_{L}^{1}(0,1):=\left\{w \in H^{1}(0,1) ; w(0)=0\right\}
$$

and endowed with the $H^{1}$-norm, and the domain

$$
\mathcal{T}:=\left\{(x, y) \in \mathbb{R}^{2} ; 0 \leq x \leq 1,0 \leq y \leq x\right\},
$$

we can state our main result.

Theorem 1: Let $\lambda \in C^{2}([0,1])$ and $\alpha, \beta \in C^{0}([0,1])$. There exist functions $h \in C^{2}([0,1])$ and $k, s \in C^{2}(\mathcal{T})$ such that for any $\left(u_{0}, u_{1}\right) \in H_{L}^{1}(0,1) \times L^{2}(0,1)$ satisfying the compatibility condition

$u_{0}(1)=\frac{1}{h(1)}\left\{\int_{0}^{1} k(1, y) u_{0}(y) d y+\int_{0}^{1} s(1, y) u_{1}(y) d y\right\}$,

there exists a unique solution of the closed-loop system (1), (3) in the space $C\left([0, \infty) ; H_{L}^{1}(0,1)\right) \cap C^{1}\left([0, \infty) ; L^{2}(0,1)\right)$. Moreover, for any $\omega>0$, there exists a positive constant $C$ independent of the initial data such that the solutions satisfy

$$
\begin{aligned}
& \left\|\left(u(\cdot, t), u_{t}(\cdot, t)\right)\right\|_{H^{1}(0,1) \times L^{2}(0,1)} \\
& \quad \leq C e^{-\omega t}\left\|\left(u_{0}, u_{1}\right)\right\|_{H^{1}(0,1) \times L^{2}(0,1)} .
\end{aligned}
$$

\section{Control Design}

In this section we derive the equations for the functions $h(x), k(x, y)$, and $s(x, y)$, and show that they have a unique twice continuously differentiable solution.

\section{A. Derivation of the equations satisfied by the kernels}

Using the transformation (2) we get

$$
\begin{aligned}
w_{t t}-w_{x x}+ & 2 d(x) w_{t}+c(x) w \\
=\int_{0}^{x} u(y)[ & k_{x x}-k_{y y}-(c(x)+\beta(y)) k \\
& -2(\lambda(y)+d(x)) s_{y y}-4 \lambda^{\prime}(y) s_{y} \\
& \left.-2\left(\lambda(y) \beta(y)+\lambda^{\prime \prime}(y)+d(x) \beta(y)\right) s\right] d y \\
+\int_{0}^{x} u_{t}(y)\left[s_{x x}-s_{y y}-2(\lambda(y)+d(x)) k\right. & \left.-\left(4 \lambda^{2}(y)+4 d(x) \lambda(y)+c(x)+\beta(y)\right) s\right] d y \\
+ & s(x, 0) u_{t x}(0)+2 u(x) \frac{d}{d x} k(x, x)+2 u(x) \lambda^{\prime}(x) s(x, x) \\
+ & u(x)\left[2(\lambda(x)+d(x)) s_{y}(x, x)\right. \\
& \left.+(c(x)+\beta(x)) h(x)-h^{\prime \prime}(x)\right] \\
+ & u_{x}(0)[k(x, 0)+2(\lambda(0)+d(x)) s(x, 0)] \\
+ & u_{t}(x)\left[2 \frac{d}{d x} s(x, x)+2(\lambda(x)+d(x)) h(x)\right] \\
- & u_{x}(x)\left[2(\lambda(x)+d(x)) s(x, x)+2 h^{\prime}(x)\right] .
\end{aligned}
$$

In order to satisfy (4), we choose $k=k(x, y)$ and $s=s(x, y)$ as solutions of

$$
\begin{aligned}
k_{x x}-k_{y y}= & 2(\lambda(y)+d(x)) s_{y y}+(c(x)+\beta(y)) k \\
& +2\left(\lambda(y) \beta(y)+\lambda^{\prime \prime}(y)+d(x) \beta(y)\right) s \\
& +4 \lambda^{\prime}(y) s_{y} \\
2 k^{\prime}(x, x)= & -2(\lambda(x)+d(x)) s_{y}(x, x)-2 \lambda^{\prime}(x) s(x, x) \\
& -(c(x)+\beta(x)) h(x)+h^{\prime \prime}(x) \\
k(x, 0)= & 0
\end{aligned}
$$

and

$$
\begin{aligned}
s_{x x}-s_{y y}= & 2(\lambda(y)+d(x)) k+(c(x)+\beta(y)) s \\
& +\left(4 \lambda^{2}(y)+4 d(x) \lambda(y)\right) s, \\
s^{\prime}(x, x)= & -(\lambda(x)+d(x)) h(x), \\
(\lambda(x)+d(x)) s(x, x)= & -h^{\prime}(x), \\
s(x, 0) & =0 .
\end{aligned}
$$

Dividing (11) by (10), we get $h^{\prime}(x) h(x)=s(x, x) s^{\prime}(x, x)$, or, integrating, $h(x)^{2}=s(x, x)^{2}+A$. Let us choose $h(0)=1$ so that when all the coefficients of the original and target systems are the same, we have the identity $w(x, t)=u(x, t)$. From (12) we have $s(0,0)=0$, which gives $A=1$. Using (11), we obtain $h^{\prime}(x) / \sqrt{h(x)^{2}-1}=\lambda(x)+d(x)$, which gives

$$
h(x)=\cosh \left(\int_{0}^{x} a(\tau) d \tau\right),
$$

where $a=a(x)$ is defined by

$$
a(x)=\lambda(x)+d(x) .
$$

Thus, we can write

$$
s(x, x)=-\frac{h^{\prime}(x)}{a(x)}=-\sinh \left(\int_{0}^{x} a(\tau) d \tau\right) .
$$


Integrating (7) and using (9) with $y=x$, after long but straightforward calculations one gets $k(x, x)$ explicitly:

$$
\begin{aligned}
k(x, x)= & \frac{h(x)}{2} \int_{0}^{x}\left(d^{2}(y)-\lambda^{2}(y)-\beta(y)-c(y)\right) d y \\
& +\frac{h^{\prime}(x)}{2 a(x)}(2 \lambda(x)+a(x)+a(0)) .
\end{aligned}
$$

Let us denote the right-hand side of the above expression by $m(x)$ and define $\rho_{i}=\rho_{i}(x, y)$ with $i=1, \ldots, 5$ by

$$
\begin{aligned}
& \rho_{1}(x, y)=2(\lambda(y)+d(x)), \quad \rho_{2}(x, y)=c(x)+\beta(y), \\
& \rho_{3}(x, y)=2 \lambda^{\prime \prime}(y)+\beta(y) \rho_{1}(x, y), \rho_{4}(x, y)=4 \lambda^{\prime}(y), \\
& \rho_{5}(x, y)=2 \lambda(y) \rho_{1}(x, y)+\rho_{2}(x, y),
\end{aligned}
$$

then one gets the following equations for the kernel functions:

$$
\begin{gathered}
\left\{\begin{aligned}
k_{x x}-k_{y y} & =\rho_{1} s_{y y}+\rho_{2} k+\rho_{3} s+\rho_{4} s_{y}, \\
k(x, x) & =m(x), \\
k(x, 0) & =0,
\end{aligned}\right. \\
\left\{\begin{aligned}
s_{x x}-s_{y y} & =\rho_{1} k+\rho_{5} s, \\
s(x, x) & =-\sinh \left(\int_{0}^{x} a(\tau) d \tau\right), \\
s(x, 0) & =0 .
\end{aligned}\right.
\end{gathered}
$$

\section{B. Existence of the kernel functions}

To prove the existence of solutions of (19), (20), we introduce the change of variables $\xi=x+y, \eta=x-y$. Denoting

$$
\begin{aligned}
G(\xi, \eta) & =k\left(\frac{\xi+\eta}{2}, \frac{\xi-\eta}{2}\right), G^{s}(\xi, \eta)=s\left(\frac{\xi+\eta}{2}, \frac{\xi-\eta}{2}\right), \\
g_{1}(\xi) & =m(\xi / 2), \quad g_{2}(\xi)=-\sinh \left(\int_{0}^{\xi / 2} a(\tau) d \tau\right), \\
b_{i}(\xi, \eta) & =\rho_{i}\left(\frac{\xi+\eta}{2}, \frac{\xi-\eta}{2}\right), \quad \forall i=1, \ldots, 4,
\end{aligned}
$$

we obtain the following PDEs from (19) and (20):

$$
\left\{\begin{aligned}
G_{\xi \eta}= & b_{1}\left(G_{\xi \xi}^{s}-2 G_{\xi \eta}^{s}+G_{\eta \eta}^{s}\right)+b_{2} G \\
& +b_{3} G^{s}+b_{4}\left(G_{\xi}^{s}-G_{\eta}^{s}\right) \\
G(\xi, 0)= & g_{1}(\xi) \\
G(\xi, \xi)= & 0
\end{aligned}\right.
$$

and

$$
\left\{\begin{aligned}
G_{\xi \eta}^{s} & =b_{1} G+b_{5} G^{s}, \\
G^{s}(\xi, 0) & =g_{2}(\xi), \\
G^{s}(\xi, \xi) & =0 .
\end{aligned}\right.
$$

Integrating (21) and (22), first with respect to $\eta$ between 0 and $\eta$, and then with respect to $\xi$ between $\eta$ and $\xi$, one gets

$$
\begin{aligned}
G(\xi, \eta) & =g_{1}(\xi)-g_{1}(\eta)+F\left[G, G^{s}\right](\xi, \eta), \\
G^{s}(\xi, \eta) & =g_{2}(\xi)-g_{2}(\eta)+F^{s}\left[G, G^{s}\right](\xi, \eta),
\end{aligned}
$$

where

$$
F^{s}=\frac{1}{4} \int_{\eta}^{\xi} \int_{0}^{\eta}\left(b_{1}(\tau, s) G(\tau, s)+b_{5}(\tau, s) G^{s}(\tau, s)\right) d s d \tau
$$

and

$$
\begin{aligned}
F= & \frac{1}{4} \int_{\eta}^{\xi} \int_{0}^{\eta}\left(b_{2}(\tau, s) G(\tau, s)+b_{1}(\tau, s) G_{\xi \xi}^{s}(\tau, s)\right) d s d \tau \\
& +\frac{1}{4} \int_{\eta}^{\xi} \int_{0}^{\eta} b_{1}(\tau, s)\left(-2 G_{\xi \eta}^{s}(\tau, s)+G_{\eta \eta}^{s}(\tau, s)\right) d s d \tau \\
& +\frac{1}{4} \int_{\eta}^{\xi} \int_{0}^{\eta} b_{4}(\tau, s)\left(G_{\xi}^{s}(\tau, s)-G_{\eta}^{s}(\tau, s)\right) d s d \tau \\
& +\frac{1}{4} \int_{\eta}^{\xi} \int_{0}^{\eta} b_{3}(\tau, s) G^{s}(\tau, s) d s d \tau .
\end{aligned}
$$

We use next a classical iterative method in order to prove that the coupled equations (23)-(24) have a unique solution. Let us define the functions $G^{0}$ and $G^{s, 0}$ as

$$
G^{0}(\xi, \eta)=g_{1}(\xi)-g_{1}(\eta), \quad G^{s, 0}(\xi, \eta)=g_{2}(\xi)-g_{2}(\eta)
$$

and set up the following recursion for $n=0,1,2, \ldots$

$$
G^{n+1}=F\left[G^{n}, G^{s, n}\right], \quad G^{s, n+1}=F^{s}\left[G^{n}, G^{s, n+1}\right] .
$$

Denote $M=\max \left\{2\left\|g_{1}^{\prime}\right\|_{\infty}, 2\left\|g_{2}^{\prime}\right\|_{\infty},\left\|g_{2}^{\prime \prime}\right\|_{\infty}\right\}$, and $K=$ $(1 / 2) \max \left\{\left\|b_{1}\right\|_{C^{1}}+\left\|b_{5}\right\|_{C^{1}}, 4\left\|b_{1}\right\|_{\infty}+\left\|b_{2}\right\|_{\infty}+\left\|b_{3}\right\|_{\infty}+\right.$ $\left.2\left\|b_{4}\right\|_{\infty}\right\}$. It is straightforward to show by induction (due to space limits we omit this calculation) that for any $n \in \mathbb{N}$ we have $\left|G^{n}\right| \leq P_{n},\left|G^{s, n}\right| \leq P_{n},\left|G_{\xi}^{s, n}\right| \leq P_{n},\left|G_{\eta}^{s, n}\right| \leq P_{n}$, $\left|G_{\xi \xi}^{s, n}\right| \leq n P_{n} /(\xi+\eta),\left|G_{\eta \eta}^{s, n}\right| \leq n P_{n} /(\xi+\eta)$, where $P_{n}=M K^{n}(\xi+\eta)^{n} / n$ !. With these estimates it follows that the solutions of (23) and (24) are given by the series

$$
G(\xi, \eta)=\sum_{n=0}^{\infty} G^{n}(\xi, \eta), G^{s}(\xi, \eta)=\sum_{n=0}^{\infty} G^{s, n}(\xi, \eta),
$$

which define two continuous functions. To see that these functions are indeed more regular, we use the equations which they satisfy. From (24), we see that $G^{s}$ belongs to $C^{2}$ if $b_{1}$ and $b_{5}$ are continuous. Then, from (23), we see that if $b_{i}$ with $i=1, \ldots, 4$, are continuous functions, then $G$ belongs to $C^{2}$. Thus, we obtain the following result asserting the existence of the kernels functions $k$ and $s$.

Theorem 2: Let $\lambda \in C^{2}([0,1]), \beta \in C^{0}([0,1])$, and $d, c \in$ $C^{0}([0,1])$. Then the equations (19) and (20) have a unique solution $k, s \in C^{2}(\mathcal{T})$.

\section{Stability of the Target System}

The exponential stability of the target system (4) has been studied by Cox and Zuazua in [4] in the case $c=0$ and by Shubov in [14] in the general case, considering even a non-constant diffusive coefficient. Their approach is spectral: they prove that the eigenfunctions of the underlying nonself-adjoint operator form a Riesz basis of the space and that the best exponential decay rate is exactly given by $\sup _{k \in \mathbb{N}} \Re\left(\sigma_{k}\right)$, where the set $\left\{\sigma_{k}\right\}_{k \in \mathbb{N}}$ is the set of eigenvalues of the stationary operator and $\Re(z)$ stands for the real part of a complex number $z$. The result in [4] is the following.

Theorem 3 ([4]): There exist two positive constants $C, \omega$ such that for any $\left(w_{0}, w_{1}\right) \in H_{0}^{1}(0,1) \times L^{2}(0,1)$, the solution of (4) satisfies

$$
\left\|\left(w(\cdot, t), w_{t}(\cdot, t)\right)\right\|_{H} \leq C e^{-\omega t}\left\|\left(w_{0}, w_{1}\right)\right\|_{H} \quad \forall t>0 .
$$


Since the functions $d$ and $c$ are part of the design of the feedback law, we are able to consider the equation (4) with constant coefficients. For this case, for any $\omega>0$, we can find the parameters $d$ and $c$ such that (26) holds, i.e arbitrary decay rate is achieved.

\section{Stability of the Closed-Loop System}

Let us define the map

$$
\begin{aligned}
\Pi: H_{L}^{1}(0,1) \times L^{2}(0,1) & \longrightarrow H_{L}^{1}(0,1) \times L^{2}(0,1), \\
\left(q_{1}, q_{2}\right) & \Pi\left(q_{1}, q_{2}\right)=\left(z_{1}, z_{2}\right),
\end{aligned}
$$

where $z_{1}, z_{2}$ are defined by

$$
\begin{aligned}
z_{1}(x):= & h(x) q_{1}(x)-\int_{0}^{x}\left[k(x, y) q_{1}(y)+s(x, y) q_{2}(y)\right] d y, \\
z_{2}(x):= & s_{y}(x, x) q_{1}(x)-s(x, x) q_{1}^{\prime}(x)+h(x) q_{2}(x) \\
& -\int_{0}^{x}[\lambda(y) s(x, y)+k(x, y)] q_{2}(y) d y \\
& -\int_{0}^{x}\left[\beta(y) s(x, y)+s_{y y}(x, y)\right] q_{1}(y) d y .
\end{aligned}
$$

This linear map is continuous and hence there exists a positive constant $D_{1}$ such that

$$
\left\|\Pi\left(q_{1}, q_{2}\right)\right\|_{H} \leq D_{1}\left\|\left(q_{1}, q_{2}\right)\right\|_{H},
$$

where $H=H^{1}(0,1) \times L^{2}(0,1)$. From Sections II-IV, it is easy to see that for any $u_{0} \in H_{L}^{1}(0,1)$ and $u_{1} \in L^{2}(0,1)$, satisfying the compatibility condition

$$
u_{0}(1)=\frac{\int_{0}^{1} k(1, y) u_{0}(y) d y+\int_{0}^{1} s(1, y) u_{1}(y) d y}{\cosh \left(\int_{0}^{1} a(\tau) d \tau\right)},
$$

one gets $\left(w_{0}, w_{1}\right)=\Pi\left(u_{0}, u_{1}\right) \in H_{0}^{1}(0,1) \times L^{2}(0,1)$, in other words, $\Pi$ maps the closed-loop system into the target system. Note that this map is invertible. Indeed, to obtain the kernels functions $\hat{k}=\hat{k}(x, y)$ and $\hat{s}=\hat{s}(x, y)$ defining $\Pi^{-1}$, we simply replace the functions $d(x)$ by $-\lambda(x)$ and $\lambda(x)$ by $-d(x)$ in the previous analysis for the kernels $k=k(x, y)$ and $s=s(x, y)$. Thus, we get a map

$$
\Pi^{-1}: H_{L}^{1}(0,1) \times L^{2}(0,1) \longrightarrow H_{L}^{1}(0,1) \times L^{2}(0,1)
$$

and a positive constant $D_{2}$ such that

$$
\left\|\Pi^{-1}\left(z_{1}, z_{2}\right)\right\|_{H} \leq D_{2}\left\|\left(z_{1}, z_{2}\right)\right\|_{H} .
$$

For $t>0$ we have that $\left(u(t), u_{t}(t)\right):=\Pi^{-1}\left(w(t), w_{t}(t)\right)$ is the solution of the closed-loop system

$$
\left\{\begin{array}{l}
u_{t t}=u_{x x}+2 \lambda(x) u_{t}+\alpha(x) u_{x}+\beta(x) u, \\
u(0, t)=0, \quad u(1, t)=\frac{\int_{0}^{1} k(1, y) u(y, t) d y+\int_{0}^{1} s(1, y) u_{t}(y, t) d y}{\cosh \left(\int_{0}^{1} a(\tau) d \tau\right)}, \\
u(x, 0)=u_{0}(x), \quad u_{t}(x, 0)=u_{1}(x),
\end{array}\right.
$$

For $\left(w_{0}, w_{1}\right) \in H_{0}^{1}(0,1) \times L^{2}(0,1)$ we have that $w \in$ $C\left([0, \infty) ; H_{0}^{1}(0,1)\right) \cap C^{1}\left([0, \infty) ; L^{2}(0,1)\right)$, the unique solution of (4), satisfies (see Theorem 3 )

$$
\left\|\left(w(\cdot, t), w_{t}(\cdot, t)\right)\right\|_{H} \leq C e^{-\omega t}\left\|\left(w_{0}, w_{1}\right)\right\|_{H} \quad \forall t>0 .
$$

From (27) and (28) we get

$$
\left\|\left(u(\cdot, t), u_{t}(\cdot, t)\right)\right\|_{H} \leq D_{2} C D_{1} e^{-\omega t}\left\|\left(u_{0}, u_{1}\right)\right\|_{H},
$$

which completes the proof of Theorem 1.

\section{Closed Form Controllers}

In this section we present several explicit control designs.

\section{A. Undamped wave equation}

Let $\lambda \equiv 0, \beta \equiv 0$ in (1):

$$
\left\{\begin{array}{l}
u_{t t}(x, t)=u_{x x}(x, t) \\
u(0, t)=0 \\
u(1, t)=U(t) .
\end{array}\right.
$$

This wave equation has all of its eigenvalues on the imaginary axis. Let us move all of them to the left in the complex plane by the same distance, parallel to the real axis (in other words, only real parts of the eigenvalues are changed). This corresponds to selecting the "critically damped" target system, a special case of (4) with $c=d^{2}$ :

$$
\left\{\begin{array}{l}
w_{t t}(x, t)=w_{x x}(x, t)-2 d w_{t}(x, t)-d^{2} w(x, t) \\
w(0, t)=0 \\
w(1, t)=0
\end{array}\right.
$$

All of the eigenvalues of the above system lie on the vertical line $\Re\left\{\sigma_{k}\right\}=-d$, which is easy to see by using the transformation $w=e^{-d t} v$, and showing that $v$ satisfies the undamped wave equation.

The PDEs (19) and (20) become

$$
\left\{\begin{array}{l}
k_{x x}(x, y)=k_{y y}(x, y)+2 d s_{y y}(x, y) \\
k(x, 0)=0 \\
k(x, x)=d \sinh (d x)
\end{array}\right.
$$

and

$$
\left\{\begin{array}{l}
s_{x x}(x, y)=s_{y y}(x, y)+2 d k(x, y) \\
s(x, 0)=0 \\
s(x, x)=-\sinh (d x)
\end{array}\right.
$$

The form of boundary conditions in the above PDEs suggests

$$
k(x, y)=d \sinh (d y), \quad s(x, y)=-\sinh (d y)
$$

as a guess for a solution. Substituting these functions into the PDEs (31), (32) we confirm that (33) is indeed a (unique) solution.

The transformation (2) can now be written as

$$
\begin{aligned}
w(x, t)= & \cosh (d x) u(x, t) \\
& +\int_{0}^{x} \sinh (d y)\left(u_{t}(y, t)-d u(y, t)\right) d y
\end{aligned}
$$

and the controller is

$$
U(t)=-\int_{0}^{1} \frac{\sinh (d y)}{\cosh (d)}\left(u_{t}(y, t)-d u(y, t)\right) d y .
$$

B. "Perfect" Lyapunov function for passively damped wave equation

Consider the plant

$$
\left\{\begin{array}{l}
u_{t t}(x, t)=u_{x x}(x, t) \\
u(0, t)=0 \\
u_{x}(1, t)=U(t)
\end{array}\right.
$$

It is well known that a so-called passive damper $U(t)=$ $-c_{1} u_{t}(1, t), c_{1}>0, c_{1} \neq 1$, exponentially stabilizes this 
system. Let us see what the backstepping design gives for this plant. We use the transformation (34) and the following target system:

$$
\left\{\begin{array}{l}
w_{t t}(x, t)=w_{x x}(x, t)-2 d w_{t}(x, t)-d^{2} w(x, t) \\
w(0, t)=0 \\
w_{x}(1, t)=0 .
\end{array}\right.
$$

From (34) it is easy to see that the controller is

$$
U(t)=-\tanh (d) u_{t}(1, t),
$$

so we recover the classical passive damper with $c_{1}=$ $\tanh (d)$. This is not surprising, because our design moves eigenvalues to the left parallel to the real axis (since (37) is critically damped) and that is also exactly what passive damper is known to do. To put it another way, we found the similarity transformation (34) between the plant with boundary damping and the plant with internal damping (critically damped). The benefit of that similarity transformation is that for the system (37) it is much easier to come up with the Lyapunov function that shows arbitrary decay rate. In fact, the simple Lyapunov function

$$
V=\frac{1}{2} \int_{0}^{1}\left(w_{t}+d w\right)^{2} d x+\frac{1}{2} \int_{0}^{1} w_{x}^{2} d x
$$

gives $\dot{V}=-2 d V$, which is the exact decay rate given by the eigenvalues (hence one can call this Lyapunov function "perfect" in some sense).

Using the transformation

$$
\begin{aligned}
u(x, t)= & \cosh (d x) w(x, t) \\
& -\int_{0}^{x} \sinh (d y)\left(w_{t}(y, t)+2 d w(y, t)\right) d y,
\end{aligned}
$$

which is inverse to (34), we rewrite the above Lyapunov function in the original variables. After simple calculations one gets

$V=\frac{1}{4} \int_{0}^{1} e^{2 d x}\left(u_{t}+u_{x}\right)^{2} d x+\frac{1}{4} \int_{0}^{1} e^{-2 d x}\left(u_{t}-u_{x}\right)^{2} d x$.

To the best of our knowledge, such a Lyapunov function (which shows the precise decay rate given by eigenvalues) does not exist in the previous literature on this classical problem. It resembles the one in [3] for the first order hyperbolic equations, however, the control design in [3] is different (passive dampers on both ends for two transport PDEs interconnected through boundaries) and the best decay rate is not shown.

Given that $c_{1}=\tanh (d)<1$ for all $d>0$, it may appear that the design above recovers passive damper only for $0<c_{1}<1$, while it is known that $c_{1}>1$ also works. However, simply modifying the boundary condition of the target system (37) at $x=1$ to the dynamic boundary condition $w_{t}(1, t)+d w(1, t)=0$ (which shifts eigenvalues vertically by $\pi / 2$ ), and using the transformation (34), we get $U(t)=-\operatorname{coth}(d) u_{t}(1, t), c_{1}=\operatorname{coth}(d)>1$. The Lyapunov function (39) with $d=\operatorname{coth}^{-1}\left(c_{1}\right)$ gives $\dot{V}=-2 d V$.
C. Assignment of arbitrary damping and stiffness for critically anti-damped wave equation

Consider the plant

$$
\left\{\begin{array}{l}
u_{t t}(x, t)=u_{x x}(x, t)+2 \lambda u_{t}(x, t)-\lambda^{2} u(x, t) \\
u(0, t)=0 \\
u(1, t)=U(t) .
\end{array}\right.
$$

All eigenvalues of this plant lie on the vertical line $\Re\left\{\sigma_{k}\right\}=$ $\lambda$. We assign arbitrary damping and stiffness using a two-step design.

Step 1: Transform the plant into the critically damped system (30). This corresponds to moving all eigenvalues to the left by $(\lambda+d)$. The PDEs for $k$ and $s$ are

$$
\left\{\begin{aligned}
k_{x x}-k_{y y} & =2(\lambda+d)\left(s_{y y}-\lambda^{2} s\right)+\left(d^{2}-\lambda^{2}\right) k \\
k(x, 0) & =0 \\
k(x, x) & =(2 \lambda+d) \sinh ((d+\lambda) x)
\end{aligned}\right.
$$

and

$$
\left\{\begin{aligned}
s_{x x}-s_{y y} & =2(\lambda+d) k+\left(3 \lambda^{2}+4 \lambda d+d^{2}\right) s \\
s(x, 0) & =0 \\
s(x, x) & =-\sinh ((\lambda+d) x) .
\end{aligned}\right.
$$

As in Section VI-A, based on the boundary conditions we take the following guess:

$$
\begin{aligned}
& k(x, y)=(2 \lambda+d) \sinh ((\lambda+d) y), \\
& s(x, y)=-\sinh ((\lambda+d) y) .
\end{aligned}
$$

One can then verify that this pair of functions is indeed a solution of the PDEs (42), (43).

Step 2: Adjust the stiffness coefficient to the desired level. We use the transformation

$$
\bar{w}(x, t)=w(x, t)-\int_{0}^{x} p(x, y) w(y, t) d y,
$$

to convert (30) into the system

$$
\left\{\begin{array}{l}
\bar{w}_{t t}(x, t)=\bar{w}_{x x}(x, t)-2 d \bar{w}_{t}(x, t)-c \bar{w}(x, t) \\
\bar{w}(0, t)=0 \\
\bar{w}(1, t)=0 .
\end{array}\right.
$$

One can show that $p(x, y)$ satisfies

$$
\left\{\begin{array}{l}
p_{x x}(x, y)=p_{y y}(x, y)+\left(c-d^{2}\right) p(x, y) \\
p(x, 0)=0 \\
p(x, x)=-\frac{1}{2}\left(c-d^{2}\right) x .
\end{array}\right.
$$

The solution to this PDE is [15]

$$
p(x, y)=-\left(c-d^{2}\right) y \frac{I_{1}\left(\sqrt{\left(c-d^{2}\right)\left(x^{2}-y^{2}\right)}\right)}{\sqrt{\left(c-d^{2}\right)\left(x^{2}-y^{2}\right)}},
$$

where $I_{1}$ is the modified Bessel function of order one.

To find the total transformation from $u$ to $\bar{w}$, we combine the transformations (2) and (46) to get

$$
\begin{aligned}
\bar{w}(x, t)= & \cosh ((\lambda+d) x) u(x, t)-\int_{0}^{x} \bar{k}(x, y) u(y, t) d y \\
& -\int_{0}^{x} \bar{s}(x, y) u_{t}(y, t) d y
\end{aligned}
$$


where

$$
\begin{gathered}
\bar{s}(x, y)=-I_{0}\left(\sqrt{\left(c-d^{2}\right)\left(x^{2}-y^{2}\right.}\right) \sinh ((\lambda+d) y) \\
\bar{k}(x, y)=(2 \lambda+d) I_{0}\left(\sqrt{\left(c-d^{2}\right)\left(x^{2}-y^{2}\right)}\right) \sinh ((\lambda+d) y) \\
-\left(c-d^{2}\right) y \frac{I_{1}\left(\sqrt{\left(c-d^{2}\right)\left(x^{2}-y^{2}\right)}\right)}{\sqrt{\left(c-d^{2}\right)\left(x^{2}-y^{2}\right)}} \cosh ((\lambda+d) y) .
\end{gathered}
$$

The feedback law is given by

$$
U(t)=\frac{\int_{0}^{1} \bar{k}(1, y) u(y, t) d y+\int_{0}^{1} \bar{s}(1, y) u_{t}(y, t) d y}{\cosh (\lambda+d)}
$$

\section{Plant with "pure" anti-damping}

For the plant

$$
u_{t t}(x, t)=u_{x x}(x, t)+2 \lambda u_{t}(x, t)
$$

the two-step approach described above gives the following gains for controller (3):

$$
\begin{aligned}
s(x, y)= & \sinh ((\lambda+d) y)+\lambda y r(x, y) \\
k(x, y)= & -\lambda y \cosh ((\lambda+d) x) \frac{I_{1}\left(\lambda \sqrt{x^{2}-y^{2}}\right)}{\sqrt{x^{2}-y^{2}}} \\
& -(2 \lambda+d)[\sinh ((\lambda+d) y)+\lambda y r(x, y)]
\end{aligned}
$$

where

$$
r(x, y)=\int_{y}^{x} \sinh ((\lambda+d) \xi) \frac{I_{1}\left(\lambda \sqrt{\xi^{2}-y^{2}}\right)}{\sqrt{\xi^{2}-y^{2}}} d \xi
$$

\section{EXTENSIONS}

The control design presented in this paper allows several straightforward extensions.

\section{A. Neumann actuation}

To extend the design to the plants with Neumann actuation we modify one of the boundary conditions of the target system (4) from $w(1, t)=0$ to $w_{x}(1, t)=0$. Using the exact same transformation (2) then gives the following feedback

$$
\begin{aligned}
u_{x}(1, t) & =\frac{1}{h(1)}\left[\left(-h^{\prime}(1)+k(1,1)\right) u(1, t)+s(1,1) u_{t}(1, t)\right. \\
& \left.+\int_{0}^{1} k_{x}(1, y) u(y, t) d y+\int_{0}^{1} s_{x}(1, y) u_{t}(y, t) d y\right] .
\end{aligned}
$$

\section{B. Robin boundary condition at the uncontrolled end}

For plants with the boundary condition $u_{x}(0, t)=$ $-q u(0, t)$ instead of the Dirichlet $u(1, t)=0$ the transformation (2) leads to the PDEs (19), (20) with boundary conditions modified as follows:

$$
\begin{aligned}
k(x, x) & =m(x)+q, \quad k_{y}(x, 0)=-q k(x, 0), \\
s_{y}(x, 0) & =-q s(x, 0),
\end{aligned}
$$

and the same boundary condition for $s(x, x)$. Using the method of successive approximations with very slight modifications compared to Section III-B, one proves existence and uniqueness of the solution of the control gain PDEs.

\section{In-domain boundary and integral terms}

The transformation (2) also works for the class of plants

$$
\begin{aligned}
u_{t t}= & u_{x x}+2 \lambda(x) u_{t}+\beta(x) u+g_{1}(x) u(0, t) \\
& +g_{2}(x) u_{x}(0, t)+\int_{0}^{x} f(x, y) u(y, t) d y,
\end{aligned}
$$

which may appear as a part of the design for more complex systems. The extra terms here are strict-feedback and therefore do not pose any difficulties for the backstepping design.

\section{Observers and output feedback}

In the designs in previous sections we assumed the measurements of $u$ and $u_{t}$ across the domain. Using the ideas presented in [16], it is possible to design dual observers which require only boundary measurements of $u$ and $u_{t}$, either on the same or on the opposite boundary with actuation. These observers can then be combined with the backstepping controllers using the certainty equivalence principle.

\section{REFERENCES}

[1] C. Bardos, G. Lebeau, and J. Rauch, "Sharp sufficient conditions for the observation, control, and stabilization of waves from the boundary," SIAM J. Control Optim., vol. 30, pp. 1024-1065, 1992.

[2] G. Chen, "Energy decay estimates and exact boundary value controllability for the wave equation in a bounded domain," J. Math. Pures Appl., vol. 58, pp. 249-273, 1979.

[3] J. M. Coron, B. d'Andréa-Novel, and G. Bastin, "A Strict Lyapunov Function for Boundary Control of Hyperbolic Systems of Conservation Laws," IEEE Trans. on Automatic Control, vol. 52, pp. 2-11, 2007.

[4] S. Cox, and E. Zuazua, "The rate at which energy decays in a damped string," Comm. Partial Diff. Equations, vol. 19, pp. 213-243, 1994.

[5] V. Komornik, Exact controllability and stabilization, RAM: Research in Applied Mathematics, Masson, Paris, 1994.

[6] V. Komornik, "Rapid boundary stabilization of linear distributed systems," SIAM J. Control Optim., vol. 35, pp. 1591-1613, 1997.

[7] V. Komornik, and P. Loreti, Fourier series in control theory, Springer Monographs in Mathematics, Springer-Verlag, New York, 2005.

[8] M. Krstic, B. Z. Guo, A. Balogh, and A. Smyshlyaev, "Outputfeedback stabilization of an unstable wave equation," Automatica, vol. 44, pp. 63-74, 2008.

[9] J. Lagnese, "Decay of solutions of wave equations in a bounded region with boundary dissipation," J. Diff. Eq., vol. 50, pp. 163-182, 1983.

[10] I. Lasiecka, and R. Triggiani, "Riccati equations for hyperbolic partial differential equations with $L_{2}\left(0, T ; L_{2}(\Gamma)\right)$-Dirichlet boundary terms," SIAM J. Control Optim., vol. 24, pp. 884-925, 1986.

[11] J.-L. Lions, Contrôlabilité exacte, perturbations et stabilisation de systèmes distribués, Masson, Paris, 1988.

[12] W. Liu, "Boundary feedback stabilization of an unstable heat equation," SIAM J. Control Optim., vol. 42, pp. 1033-1043, 2003.

[13] D. L. Russell, "Controllability and stabilizability theory for linear partial differential equations: Recent progress and open questions," SIAM Rev., vol. 20, pp. 639-739, 1978.

[14] M. A. Shubov, "Nonselfadjoint operators generated by the equation of a nonhomogeneous damped string," Trans. Amer. Math. Soc., vol. 349, pp. 4481-4499, 1997.

[15] A. Smyshlyaev, and M. Krstic, "Closed-form boundary state feedbacks for a class of 1-D partial integro-differential equations," IEEE Trans. Automat. Control, vol. 49, pp. 2185-2202, 2004.

[16] A. Smyshlyaev, and M. Krstic, "Backstepping observers for a class of parabolic PDEs," Syst. Control Lett., vol. 54, pp. 613-625, 2005.

[17] A. Smyshlyaev, and M. Krstic, "Boundary control of an anti-stable wave equation with anti-damping on the uncontrolled boundary," Syst. Control Lett., vol. 58, pp. 617-623, 2009.

[18] M. Tucsnak, "Boundary stabilization for the stretched string equation," Differential Integral Equations, vol. 6, pp. 925-935, 1993.

[19] J. M. Urquiza, "Rapid exponential feedback stabilization with unbounded control operators," SIAM J. Control Optim., vol. 43, pp. 2233-2244, 2005. 